\section{Elucidating molecular connetion between IAHSP onset and Alsin protein by means of Homology Modelling and Molecular Dynamics}

\author{
Marcello Miceli, ${ }^{1}$ Cecile Exertier, ${ }^{2}$ \\ Vallone Beatrice,2 Marco Cavaglià, 1 \\ Marco A. Deriu1 \\ 1PolitoBIOMedLab, Department of \\ Mechanical and Aerospace Engineering, \\ Politecnico di Torino,Turin; \\ 2Dipartimento di Scienze Biochimiche \\ “A. Rossi Fanelli", Sapienza, Università \\ di Roma, Rome, Italy
}

\begin{abstract}
The Infantile-onset Ascending Hereditary Spastic Paralysis (IAHSP) is an incurable rare neurodegerative disease related to a mutation-driven aberrant behaviour of the Alsin protein. The lack of information on Alsin atomic structure limits a complete understanding on pathology mechanisms. In this work, molecular modelling techniques have been applied to shed lights on Alsin folding dynamics and misfunction induced by aberrant mutations.
\end{abstract}

\section{Introduction}

Alsin is a key protein involved in the onset of the IAHSP, a rare pathology characterized by the degeneration of upper motor neurons of the pyramidal tract. The Alsin protein, encoded by the Als 2 gene, ${ }^{1}$ is composed of 1657 amino acids and also expressed in the spinal cord and brain. ${ }^{2}$ Alsin is predicted as formed by three structured domains, i.e.: i) the RCC1-like domain (RLD) in N-terminal position, ii) the central B cell lymphomahomology (DH) and pleckstrin-homology (PH) domain, and iii) the C-terminal vacuolar protein sorting 9 (VPS9) domain. In-vitro studies have proposed that a crucial step for the Alsin physiological pathway is the selftetramerization and has highlighted how single point mutations in the sequence of VPS9 structured domain are correlated to a reduced tendency to oligomerise ${ }^{3}$ and thus to the onset of the pathology. Nowadays, any resolved protein model for the Alsin is available, limiting computational studies and computer-aided drug discovery investigations. For this reason, the current research will be focused on the development of a homology model for the VPS9 domain of Alsin. The point mutation $\mathrm{R} 1611 \mathrm{~W}$ in VPS9, i.e. amino acid change in position 1611, Arginine to Tryptophane, altering Alsin function, will be investigated. Models developed will be employed to understand the effect of single point mutation on the stability of the structured domain and to suggest possible alteration on the selfoligomerization process.

\section{Materials and Methods}

To predict the secondary and tertiary structure, the VPS9 sequence obtained from the Uniprot database (Q96Q42) has been employed. Homology modelling has been performed through the I-Tasser and the quality of the model has been assessed with PROCHECK software. Subsequently, the obtained model of the VPS9 Wild Type (WT) has been mutated to obtain a model for the single point mutant R1611W (MUT). Three replicas of $1.5 \mu$ s long Molecular Dynamics (MD) simulations have been carried out both for the WT and MUT systems. All systems' topology has been modelled through AMBER99sb-ildn forcefield. Systems were placed in a dodecahedron box filled with explicit water (TIP3P) and neutralized with $\mathrm{Na}^{+}$and $\mathrm{Cl}^{-}$ ions added at a physiological concentration of $0.15 \mathrm{M}$. The engine employed for MD simulations and analysis was GROMACS 2020.3. Firstly temperature $(298 \mathrm{~K})$ and pressure (1 bar) have been equilibrated restraining protein positions. After removing restraints the MD production was performed at constant temperature $(298 \mathrm{~K})$ and pressure (1 bar). The radius of gyration $(R g)$ has been employed as a measure of the compactness of the structure. Results have been obtained as the time average concatenating the last $500 \mathrm{~ns}$ of simulations.

\section{Results}

The quality of VPS9 homology model (Figure 1A) has been evaluated through ITasser scores, i.e. the Confidence Score (CScore) and Template Modelling Score (TMScore) respectively equal to 1.76 and $0.96 \pm 0.05$. Moreover, the stereochemistry has been validated via the Ramachandran plot, which reported $95,6 \%$ of residues in the most favoured regions and $4,6 \%$ in the additional allowed regions (Figure 1B).

Molecular dynamics simulation both on the WT and the MUT models have been done to understand the consequences of the mutation on the protein function. Figure 2
Correspondence: Marcello Miceli, PolitoBIOMedLab, Department of Mechanical and Aerospace Engineering, Politecnico di Torino, Turin, Italy.

E-mail: marcello.miceli@polito.it

Key words: Molecular dynamics; homology modelling; neurodegenerative; IAHSP; rare pathologies.

Acknowledgments: This work has been financed in the framework of the Telethon project CRYSTAL (GA: GSP20005_PAsIAHSP007).

Disclosures: The authors have declared no conflicts of interest

Conference presentation: This paper was presented at the Third Centro 3R Annual Meeting - L'era delle 3R: modelli in silico, in vitro e in vivo per promuovere la ricerca traslazionale 30 September - 1 October 2021, Evento online organizzato dal Politecnico di Torino.

Received for publication: 9 July 2021.

Accepted for publication: 7 September 2021.

This work is licensed under a Creative Commons Attribution NonCommercial 4.0 License (CC BY-NC 4.0).

(C) Copyright: the Author(s), 2021

Licensee PAGEPress, Italy

Biomedical Science and Engineering 2021; 4(s1):183 doi:10.4081/bse.2021.183

reports the probability distribution of $R g$ for both WT and MUT VPS9 models.

Both systems show the same mean value for the distribution of $R g$, but with remarkably different standard deviations (WT 15.4 $\pm 0.42 \AA$, MUT $15.1 \pm 0.13 \AA$ ), showing a greater ability of the MUT system to explore a wider range of values compared to the WT.

\section{Discussion and Conclusions}

The current study represents the first step on a better comprehension of the Alsin protein both developing the first homology model for the VPS9 domain and study its dynamics via Molecular Modelling techniques. Computational results pointed attention to conformational modifications and flexibility changes in protein dynamics, induced by the R1611W mutation. Indeed, as observed in vitro, mutated forms of Alsin shows a reduced tendency to oligomerize. Being the $R g$ a measure of protein compactness, results suggest that the Wild Type (WT) can explore a broader range of conformations than the Mutated (MUT), sug- 


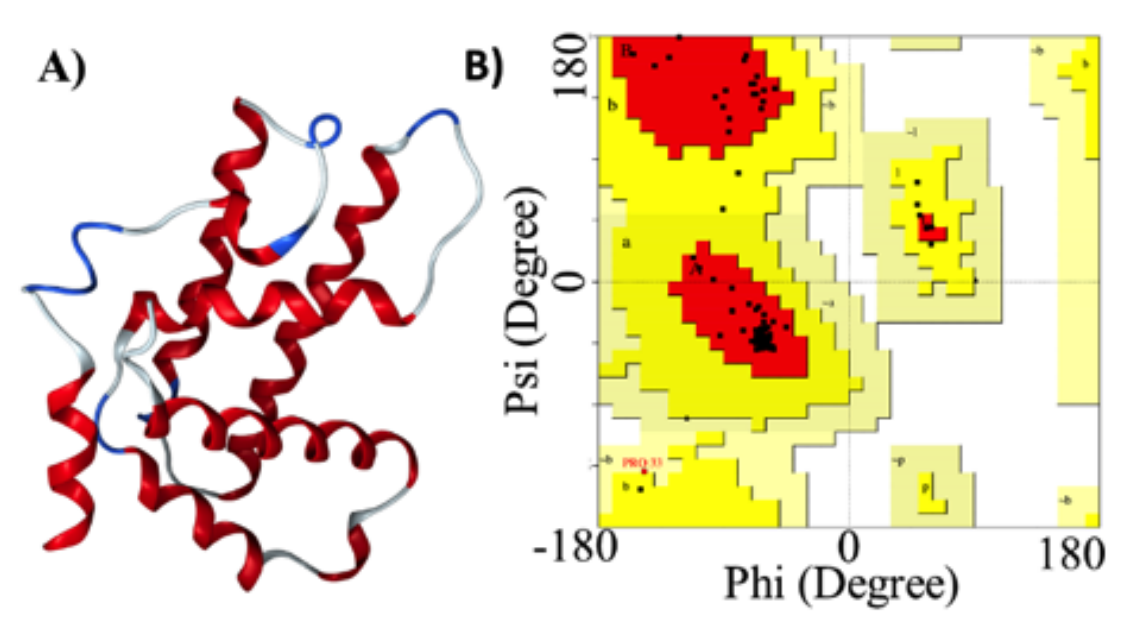

Figure 1. A) I-Tasser Homology model for the VPS9 (structured domain showed in New Cartoon representation); B) Rhamacandran Plot.

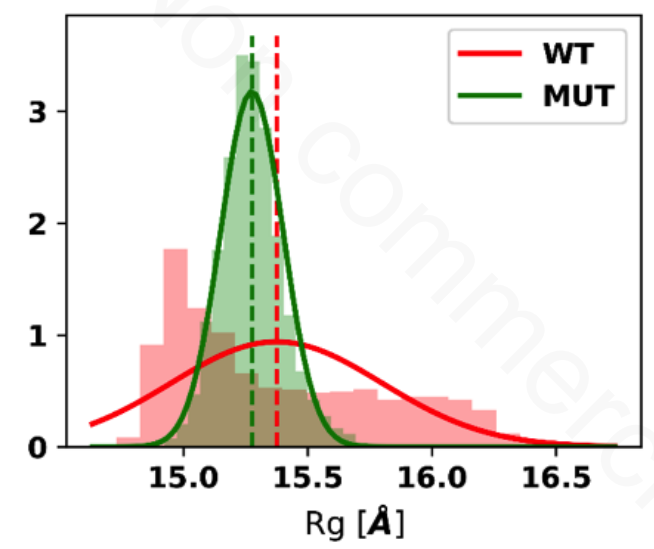

Figure 2. Probability density for Radius of Gyration for the WT (red) and the MUT (Green). gesting for increased stiffness for the MUT form. This result can be correlated to the reduced tendency of MUT to the selfoligomerization associated to a lowered ability to rearrange its conformation and thus to be able to be engaged for proteinprotein interaction and to its natural pathway. This altered bheaviour can be a possible explanation on the involvement of this molecule in the onset of the IAHSP. This result with the first 3D model of VPS9 developed can be a starting point for future drug screening studies aimed at restoring the physiological function of Alsin and for a better comprehension of the onset of IAHSP and Alsin-related pathologies.

\section{References}

1. Hadano S, Hand CK, Osuga H, et al. A gene encoding a putative GTPase regulator is mutated in familial amyotrophic lateral sclerosis 2. Nat Genet 2001;29: 166-73.

2. Yang Y, Hentati A, Deng HX, et al. The gene encoding alsin, a protein with three guanine-nucleotide exchange factor domains, is mutated in a form of recessive amyotrophic lateral sclerosis. Nat Genet 2001;29:160-5.

3. Sato K, Otomo A, Ueda MT, Altered oligomeric states in pathogenic ALS2 variants associated with juvenile motor neuron diseases cause loss of ALS2mediated endosomal functionet al. J Biol Chem 2018;293:17135-53. 